\title{
Canonical Methods in the Solution of Variable- Coefficient Lanchester-Type Equations of Modern Warfare
}

\author{
JAMES G. TAYLOR and GERALD G. BROWN \\ Naval Postgraduate School, Monterey, California
}

(Received original October 17, 1974; final, May 12, 1975)

\begin{abstract}
This paper develops a mathematical theory for solving deterministic, Lanchester-type, 'square-law' attrition equations for combat between two homogeneous forces with temporal variations in fire effectivenesses (as expressed by the Lanchester attrition-rate coefficients). It gives a general form for expressing the solution of such variablecoefficient combat attrition equations in terms of Lanchester functions, which are introduced here and can be readily tabulated. Different Lanchester functions arise from different mathematical forms for the attrition-rate coefficients. We give results for two such forms: (1) effectiveness of each side's fire proportional to a power of time, and (2) effectiveness of each side's fire linear with time but with a nonconstant ratio of attrition-rate coefflcients. Previous results in the literature for a nonconstant ratio of these attrition-rate coefficients only took a convenient form under rather restrictive conditions.
\end{abstract}

$D^{\mathrm{n}}$

ETERMINISTIC Lanchester-type equations of warfare (see references 38,44 ) are of value for identifying trends in weapon system analysis or force structuring studies because of their computational convenience, even though combat between two opposing military forces is indeed a complex random process (see Note 1). In this paper we present a mathematical theory (including a new standard form) for the solution of variable-coeffcient Lanchester-type equations of modern warfare for combat between two homogeneous forces. After some preliminaries, we give a more precise statement of this paper's purpose at the end of Section 3.

First, we review Lanchester's classic mathematical model of combat between two homogeneous forces and its extension to cases of time-varying fire effectiveness. Then we discuss previous work on developing analytic solutions to variable-coefficient Lanchester-type equations and explain how we extend these results. Next, we develop a mathematical theory for solving Lanchester-type equations for a 'square-law' attrition process for combat between two homogeneous forces. These general results are then ap- 
plied to cases of some particular attrition-rate coefficients of interest, and new mathematical functions, Lanchester functions, that could be tabulated in the future are proposed. Some numerical examples are given. Finally, we discuss the development of tabulations of values of Lanchester functions.

\section{LANCHESTER'S CLASSIC FORMULATION}

F. W. Lanchester was an English aeronautical engineer who lived from 1868 to 1946 (see reference 25 ). In 1914 he hypothesized in reference 23 that combat between two opposing forces could be modelled by (see Note 2)

$$
\begin{array}{lll}
d x / d t=-a y & \text { with } & x(t=0)=x_{0}, \\
d y / d t=-b x & \text { with } & y(t=0)=y_{0},
\end{array}
$$

where $t=0$ denotes the time at which the battle begins, $x(t)$ and $y(t)$ denote the numbers of $X$ and $Y$ at time $t$, and $a$ and $b$ are nonnegative constants, which are today called Lanchester attrition-rate coefficients. These coefficients represent the effectiveness of each side's fire (i.e., its firepower). Lanchester considered his now classic combat formulation (1) in order to provide insight into the dynamics of combat under "modern conditions" and justify the principle of concentration (see Note 3 ). The above equations are valid only for $x, y>0$. The first, for example, becomes $d x / d t=0$ for $x=0$.

We shall refer to the equations (1) as Lanchester's equations of modern warfare. Two sets of circumstances under which these equations have been hypothesized to apply are:

(a) both sides use aimed fire and target acquisition times are constant, independent of the force levels (a special case is when they are negligible),

(b) both sides use area fire and a constant density defense.

A more complete discussion of these hypotheses is to be found in references 11 and 44 . Other factors may be included in the equations and other differential equation models of combat attrition may be referred to as Lanchester-type equations, but we will not consider these here (see references 15 and 38 ).

From (1) Lanchester deduced his classic square law

$$
b\left[x_{0}{ }^{2}-x^{2}(t)\right]=a\left[y_{0}{ }^{2}-y^{2}(t)\right],
$$

which has the important implication that a side can significantly reduce its casualties by initially committing more forces to battle. We also have the well known result that the time history of, for example, the $X$ force level is given by

$$
x(t)=x_{0} \cosh (a b)^{\frac{1}{2}} t-y_{0}(a / b)^{\frac{1}{2}} \sinh (a b)^{\frac{1}{2}} t .
$$


One result of this paper is a generalization of (3) to the general case of variable attrition-rate coefficients.

\section{VARIABLE ATTRITION-RATE COEFFICIENTS}

The pioneering work of Bonder ${ }^{[5,7,10]}$ on methodology for the evaluation of military systems (particularly mobile systems, such as tanks, mechanized infantry combat vehicles, etc.) provides a motivation for interest in variable-coefficient, deterministic, Lanchester-type combat formulations. Bonder ${ }^{[6]}$ has pointed out that (at least for the case of mobile weapon systems) the validity of the assumption of constant attrition-rate coefficients is open to question. Let us, therefore, consider

$$
\begin{array}{lll}
d x / d t=-a(t) y & \text { with } & x(t=0)=x_{0}, \\
d y / d t=-b(t) x & \text { with } & y(t=0)=y_{0},
\end{array}
$$

where $a(t)$ and $b(t)$ denote time-dependent Lanchester attrition-rate coefficients. Recently, Taylor and Parry have shown by consideration of the force-ratio equation that a generalization of (2) holds even for the general case of variable attrition-rate coefficients [see equation (4) of reference 41]. Accordingly, we will also refer to (4) as the equations for a 'squarelaw' attrition process (see also references 37 and 40 ).

Two significant developments in the Lanchester theory of combat during the 1960's were (a) the development of methodology for the prediction of Lanchester attrition-rate coefficients from weapon-system performance data by Bonder ${ }^{[6,8]}$ and others in references 3 and 10, and (b) Clark's ${ }^{[12]}$ development of methodology for the (maximum likelihood) estimation of such coefficients from Monte Carlo simulation output data. Both of these developments and others ${ }^{[30,31]}$ have facilitated the application for defense planning studies of models such as (4) and its generalization to combat between heterogeneous forces (see reference 10).

The Lanchester attrition-rate coefficients in (4) depend on a number of variables, such as firing doctrine, firing rate, rate of target acquisition, force separation, tactical posture of targets (see reference 5 or pp., 18-26 and pp. 81-114 of reference 10 for attrition-rate coefficient prediction methodology). Bonder ${ }^{[5,10]}$ has considered a number of forms for the range dependency of attrition-rate coefficients based on examination of data for some representative weapon systems. Motivated by this work, we will consider the following coefficients:

$$
\begin{array}{rlll}
\text { (I) } a(t)=k_{a}(t+C)^{m} & \text { and } \quad b(t)=k_{b}(t+C)^{n} \quad \text { with } \quad C \geqq 0, \\
\text { (II) } a(t)=k_{a}(t+C) & \text { and } \quad b(t)=k_{b}(t+C+A) & \text { with } & A, C \geqq 0,
\end{array}
$$

where the constant $A$ allows the ratio of attrition-rate coefficients that both vary linearly with time to be nonconstant and in (6) the constant $C$ allows 
the fire effectiveness of each of the two systems to be nonzero at the beginning of battle (i.e., $t=0$ ). In (5) the modelling role of $C$ depends on the values of $m$ and $n$; for example, if $m$ and $n>0$, then $C$ allows the initial fire effectiveness of each system to be nonzero. Some situations that may be modelled with these coefficients are discussed below. [Additionally, for $m$ and $n$ negative (for $m, n \leqq-1$ we require $C>0$ ), equations (4) and (5) may be considered to model, for example, an infantry 'fire fight' in which the combatants take cover so that the effectiveness of fires decreases with time.] For a battle in which, for example, force separation or range between two homogeneous forces changes at a constant rate, the parameters $A$ and $C$ may be related to those of range-dependent attrition-rate coefficients (see Section 7 ).

\section{PREVIOUS WORK ON DEVELOPMENT OF ANALYTIC SOLUTIONS}

Analytic solutions to Lanchester-type equations such as (4) are important, as stressed by Bonder (see pp. 30-31 of reference 10), for developing insights into the dynamics of combat by portraying the relation between various factors in the combat attrition process and the surviving numbers of forces and also for facilitating sensitivity and other parametric analyses (see reference 9). Additionally, analytic results for such variable-coefficient formulations have also proven useful in the study of the optimal control of deterministic Lanchester-type attrition processes, see references 37 , 39 , and 40 .

Oberbeck $^{[28]}$ (also see reference 33) was apparently the first to give results via successive approximations for the general case of (4). Subsequently, Bonder and Farrell ${ }^{[10]}$ and Taylor ${ }^{[38]}$ have given more explicit results, which take advantage of the structure of (4), and have applied these general results to specific functional forms of attrition-rate coefficients.

Koopman (see pp. 65-67 of reference 26) apparently first observed the important result (see Note 4) that for a constant ratio of attrition-rate coefficients the time solution to (4) takes a form no more complicated than that for constant coefficients. When

$$
a(t)=k_{a} h(t), \quad \text { and } \quad b(t)=k_{b} h(t),
$$

where $h(t)$ denotes the common time-varying factor in the two attritionrate coefficients, one obtains

$$
x(t)=x_{0} \cosh \psi(t)-y_{0}\left(k_{a} / k_{b}\right)^{\frac{1}{2}} \sinh \psi(t),
$$

where $\psi(t)=\left(k_{a} k_{b}\right)^{\frac{1}{2}} \int_{0}^{t} h(s) d s$. This result is the same as (3) except for a transformation of the time scale of battle. Subsequently, Isaacs, ${ }^{[21]}$ Farrell, ${ }^{[10]}$ and Taylor (see Appendix D of references 34 and 35) have inadvertently rediscovered this result in different modelling contexts (see reference 36 ). Solutions for special cases of (7) had been given earlier by 
Bonder. ${ }^{[5]}$ Koopman also observed that we again obtain a 'squarelaw' relation $k_{a}\left[x_{0}{ }^{2}-x^{2}(t)\right]=k_{b}\left[y_{0}{ }^{2}-y^{2}(t)\right]$.

Results are considerably more complex for a nonconstant ratio of attrition-rate coefficients. Bonder and Farrell ${ }^{[10]}$ and Taylor ${ }^{[38]}$ have given infinite series solutions for the linear attrition-rate coefficients with nonconstant ratio (6) for the special case of $C=0$ (see Note 5). Additionally, Bonder and Farrell (see pp. 296-367 of reference 10) used analogue computer methods to parametrically study the dynamics of this combat situation. Taylor ${ }^{[38]}$ has given results for the power attrition-rate coefficients (5), again for $C=0$.

Thus, all the infinite series solutions noted above for a nonconstant ratio of attrition-rate coefficients apply only to the restrictive case in which $C=0$. If we use (5) with $m, n>0$ or (6), then we are limited to modelling battles in which the fire effectiveness of at least one side is initially zero. [Within the modelling context of a constant-speed attack (see Section 7 ), the battle must begin at the minimum of the maximum effective ranges of the two systems.] The purpose of this paper is therefore to remove the requirement that $C=0$ and to present solutions in terms of new special Lanchester functions that can be tabulated. This is done for power attrition-rate coefficients with (in the context of a constant-speed attack) equal effective ranges of the combat systems and linear attrition-rate coefficients corresponding to opposing weapons with different effective ranges. Additionally, we determine what functional forms for attrition-rate coefficients lead to simplification of analytic results.

\section{A MATHEMATICAL THEORY FOR THE SOLUTION OF LANCHESTER-TYPE EQUATIONS OF MODERN WARFARE}

In this section we develop a generalization of the form of the constantcoefficient solution (3) to the general case of variable attrition-rate coefficients (see Note 6). The Lanchester-type equations (4) yield the $X$ force level equation

$$
d^{2} x / d t^{2}-\{[1 / a(t)] d a / d t\} d x / d t-a(t) b(t) x=0,
$$

with initial conditions $x(t=0)=x_{0}$, and $\{[1 / a(t)] d x / d t\}_{t=0}=-y_{0}$. The solution of (9) is given by

$$
x(t)=C_{1} x_{1}(t)+C_{2} x_{2}(t),
$$

where $\left\{x_{1}(t), x_{2}(t)\right\}$ denotes a fundamental system of solutions to $(9)$ (see p. 119 of reference 20 ), and $C_{1}$ and $C_{2}$ are constants determined by the initial conditions.

We now consider how to choose $x_{1}(t)$ and $x_{2}(t)$ so that (10) with $C_{1}$ and $C_{2}$ evaluated will be a generalization of $(3)$. We recall the well known properties of the hyperbolic functions that appear in (3)-that, for exam- 
ple, $d\left[\cosh (a b)^{\frac{1}{2}} t\right] / d t=a(b / a)^{\frac{1}{2}} \sinh (a b)^{\frac{1}{2}} t$, where $a$ and $b$ denote constants. We choose $x_{1}(t)$ and $x_{2}(t)$ so that

$$
d x_{1} / d t=k a(t) y_{2}, \quad \text { and } \quad d x_{2} / d t=k a(t) y_{1},
$$

where without further specification $k$ may be any nonzero constant. Similarly, we choose $y_{1}(t)$ and $y_{2}(t)$ to satisfy

$$
d y_{1} / d t=(1 / k) b(t) x_{2}, \text { and } d y_{2} / d t=(1 / k) b(t) x_{1} .
$$

One easily verifies that if $x_{1}(t)$ and $x_{2}(t)$ satisfy (11) and (12), then they will also satisfy the differential equation (9). Next, we consider $P(t)=$ $x_{1}(t) y_{1}(t)-x_{2}(t) y_{2}(t)$. We observe that $d P / d t$ is defined and equal to zero $\forall t \epsilon\left(t_{0},+\infty\right)$ where, assuming that $a(t)$ and $b(t)$ are both not constant,

$$
t_{0}=\max \left(t_{0}{ }^{x}, t_{0}{ }^{x}\right) \text {. }
$$

$t_{0}{ }^{x}$ denotes the largest finite singularity (see p. 160 of reference 20) on the $t$-axis for the $X$ force-level equation, and similarly for $t_{0}{ }^{Y}$ (see Note 7 ). (We take $t_{0}{ }^{x}$ to be $-\infty$ if $(9)$ has no finite singularity.) Thus, for te $\left(t_{0},+\infty\right)$ we have $P(t)=C_{N}$, where $C_{N}$ denotes a normalization constant that we may take to be equal to one without loss of generality. Hence, we can always achieve

$\forall t \epsilon\left(t_{0},+\infty\right)$.

$$
x_{1}(t) y_{1}(t)-x_{2}(t) y_{2}(t)=1
$$

The general Lanchester functions $x_{1}(t), x_{2}(t), y_{1}(t)$, and $y_{2}(t)$ may be constructed by either successive approximations (see reference 38 ) or infinite series methods (see references 20,22, and 27). Without further specifications, however, they are not uniquely determined. Moreover, if we can find four such functions satisfying (11), (12), and (14), then, for example, $x_{1}(t)$ and $x_{2}(t)$ are a fundamental system of solutions to $(9)$.

We now show that to obtain generalizations of the hyperbolic functions we must choose

$$
k=\left(k_{b} / k_{a}\right)^{\frac{1}{2}} \text {. }
$$

If $a(t)$ and $b(t)$ are both bounded at $t_{0}$, then $a\left(t=t_{0}\right) b\left(t=t_{0}\right)=0$ and $d P / d t$ $=0 \forall t \epsilon\left[t_{0},+\infty\right)$. In this case we can achieve the normalization (14) $\forall t_{\epsilon}\left[t_{0},+\infty\right)$ and uniquely determine the general Lanchester functions by choosing

$$
x_{1}\left(t=t_{0}\right)=y_{1}\left(t=t_{0}\right)=1, \quad \text { and } \quad x_{2}\left(t=t_{0}\right)=y_{2}\left(t=t_{0}\right)=0 .
$$

When (7) holds with $h(t=0)=0$ and $0<h(t)<+\infty$ for $t>0$, we may use Theorem 2 to solve (11), (12), and (16). We thus obtain

$$
x_{1}(t)=y_{1}(t)=\cosh \theta(t) \text {, and } x_{2}(t)=y_{2}(t)=\sinh \theta(t),
$$

where $\theta(t)=\left(k_{a} k_{b}\right)^{\frac{1}{2}} \int_{0}^{t} h(s) d s$. Consequently, (11) and (17) yield (15). 
Next, we show that if we choose $x_{1}(t)$ and $x_{2}(t)$ as above, then they are linearly independent. Considering the Wronskian of $x_{1}, x_{2}$, denoted as $W\left(x_{1}, x_{2}\right)$, we see that

$$
W\left(x_{1}, x_{2}\right)=\left|\begin{array}{cc}
x_{1} & x_{2} \\
\dot{x}_{1} & \dot{x}_{2}
\end{array}\right|=a(t)\left(k_{b} / k_{a}\right)^{\frac{1}{2}} .
$$

Thus, $W\left(x_{1}, x_{2}\right) \neq 0 \forall t \epsilon\left(t_{0},+\infty\right)$ (see Note 8), and the linear independence is proven.

We finally discuss how to uniquely specify $x_{1}(t), x_{2}(t), y_{1}(t)$, and $y_{2}(t)$ so that they may be constructed by, for example, infinite series methods. If $x_{1}(t), x_{2}(t), y_{1}(t)$, and $y_{2}(t)$ are bounded at $t=t_{0}$, then they are, at least in a one-sided sense, continuous at $t=t_{0}$; and we impose (16). If $x_{1}(t)$, $x_{2}(t), y_{1}(t)$, and $y_{2}(t)$ are not all bounded at $t=t_{0}$, we must do otherwise. For the Fuchsian equation (see p. 370 of reference 20 and pp. 108-

TABLE I

Properties of the General Lanchester Functions $x_{1}, x_{2}, y_{1}, y_{2}$

$\begin{array}{lr}\text { 1. } & \left.d x_{1} / d t=\left(k_{b} / k_{a}\right)\right)^{2} a(t) y_{2} \\ \text { 2. } & d x_{2} / d t=\left(k_{b} / k_{a}\right) a(t) y_{1} \\ \text { 3. } & d y_{1} / d t=\left(k_{a} / k_{b}\right) * b(t) x_{2} \\ 4 . & d y_{2} / d t=\left(k_{a} / k_{b}\right) * b(t) x_{1} \\ \text { 5. } & x_{1}(t) y_{1}(t)-x_{2}(t) y_{2}(t)=1 \quad \forall t\end{array}$

127 of reference 27$)$ that arises from the power attrition-rate coefficients (5) when $m+n+2=0$, we take $x_{1}\left(t=t_{0}\right)$ to be unbounded [so that $y_{2}\left(t=t_{0}\right)$ is also]. Then the conditions $x_{1}(t=0)=1,(11),(12)$, and (14) uniquely determine the general Lanchester functions.

The properties of the general Lanchester functions are summarized in Table I. In general, the functions $x_{1}(t)$ and $y_{1}(t)$ are like the hyperbolic cosine (with the appropriate argument), while $x_{2}(t)$ and $y_{2}(t)$ are like the hyperbolic sine. Using the specifications given in the previous paragraph, we may construct the general Lanchester functions by either successive approximations (see reference 38 ) or infinite series methods (see references $20,22,27$ ).

The constants $C_{1}$ and $C_{2}$ in (10) are determined by the initial conditions for (9), and thus by (11) and (14) it follows that

$$
\begin{array}{r}
x(t)=x_{0}\left\{y_{1}(t=0) x_{1}(t)-y_{2}(t=0) x_{2}(t)\right\} \\
-y_{0}\left(k_{a} / k_{b}\right)^{\frac{1}{2}}\left\{x_{1}(t=0) x_{2}(t)-x_{2}(t=0) x_{1}(t)\right\},
\end{array}
$$

where $a(t)=k_{a} g(t)$ and $b(t)=k_{b} h(t)$. Here $g(t)$ and $h(t)$ denote timevarying factors of $a(t)$ and $b(t)$ such that $a(t) / b(t)=k_{a} / k_{b}$ for $g(t)=h(t)$. Expression (18) is the generalization of the well known constant-coefficient 
form (3) of the $X$ force level, $x(t)$, to the general case of variable Lanchester attrition-rate coefficients. Equation (14) plays a key role in verifying that (18) does indeed satisfy the initial conditions for (9).

Recalling the well known constant ratio of attrition-rate coefficient results (8), we know that for $t_{0} \neq 0$ (18) is at least sometimes capable of further simplification. However, it is indeed remarkable that (18) can be simplified only when (7) holds.

THEOREM 1. For $t_{0} \neq 0$, one can further simplify (18) if and only if (7) holds (constant ratio of attrition-rate coefficients).

Proof. From (11) and (12) we have, for example, that $d x_{1} / d y_{2}=$ $k^{2}\{a(t) / b(t)\} y_{2} / x_{1}$. Thus a relation that is independent of $t$ exists between $x_{1}$ and $y_{2}$ if and only if $a(t) / b(t)=$ constant. Moreover, when such a relation does exist, we have by $(7)$ that

$$
\left[x_{1}(t)\right]^{2}-\left[y_{2}(t)\right]^{2}=1 \text {. }
$$

Besides being a classic Lanchester 'square law,' equation (19) is a necessary and sufficient condition, for example, for $x_{1}(t)$ to possess a so-called algebraic addition theorem (see Note 9). Unless the general Lanchester functions possess such algebraic addition theorems, for $t_{0} \neq 0$ there is no further simplification to (18).

Remark 1: When (7) does hold with $t_{0}<0$, we have (17) with $\theta(t)=$ $\left(k_{a} k_{b}\right)^{\frac{1}{2}} \int_{0}^{t} h(s) d s+\left(k_{a} k_{b}\right)^{\frac{1}{2}} \int_{t_{0}}^{0} h(s) d s$. The complementary $X$ - and $Y$-functions are identical only in this case. Moreover, (14) [or, equivalently, (19)] is then a well known property of the hyperbolic functions. Using the algebraic addition theorems for the hyperbolic functions [e.g., $\cosh u$ $\cosh v-\sinh u \sinh v=\cosh (u-v)$ ], we may simplify (18) to (8). We also note that (8) may be written as

$$
\left.x(t)=x_{0} \cosh (\overline{[a(t) b(t)}]^{\frac{1}{2}} t\right)-y_{0}[\overline{a(t) / b(t)}]^{\frac{1}{2}} \sinh \left([\overline{a(t) b(t)}]^{\frac{1}{3}} t\right),
$$

where $[\overline{a(t) b(t)}]^{\frac{1}{b}}$ denotes an average, i.e., $[\overline{a(t) b(t)}]^{\frac{1}{2}}=(1 / t) \int_{0}^{t}[a(s) b(s)]^{\frac{1}{2}} d s$. Thus, we see that in the case of a constant ratio of attrition-rate coefficients, the $X$ force level, $x(t)$, is given by an expression formally equivalent to that for the constant-coefficient case with averages being used.

Remark 2: In cases in which a square-law relation like (19) does not hold, there is no such convenient reduction of (18) with $t_{0} \neq 0$ to a simpler form like (8) via an algebraic addition theorem. Thus, in general, the $X$ force level, $x(t)$, does not take a simple form except when $t_{0}=0$ or $a(t) / b(t)$ is a constant.

Remark 3: When $t_{0}=0$, then (18) again takes a particularly simple form:

$$
x(t)=x_{0} x_{1}(t)-y_{0}\left(k_{a} / k_{b}\right)^{\frac{1}{2}} x_{2}(t) .
$$

Our previous results in reference 38 were all of the form (21).

In reference 38 (see also references 35 and 36 ) we showed that when the 
ratio of attrition-rate coefficients is constant, we can transform the $X$ forcelevel equation into one with constant coefficients by a transformation of the independent variable $t$. As we have seen, this case leads to particularly convenient results. In this respect, a useful theorem (see also Appendix $\mathrm{D}$ of reference 34 ) is

THEOREM 2. A necessary and sufficient condition to be able to transform the $X$ force-level equation (9) by a transformation of the independent variable $t$ into a linear second-order ordinary differential equation with constant coefficients is that $\{[1 / a(t)] d a / d t-[1 / b(t)] d b / d t\} /[a(t) b(t)]^{\frac{3}{2}}$ is a constant.

Proof. The theorem follows immediately from a result given on pp. 7374 of reference 4 : the equation $d^{2} x / d t^{2}+p(t) d x / d t+q(t) x=0$ can be transformed to a linear equation with constant coefficients by a transformation of the independent variable alone if and only if the expression $\{(d q / d t+$ $\left.2 p q) / q^{3 / 2}\right\}$ is found to be constant.

Moreover, when the $X$ force-level equation is so transformable, the desired substitution is given by $u=K \int^{t}[a(s) b(s)]^{\frac{1}{2}} d s$, where $\int^{t} \cdots d s$ denotes an indefinite integral and $K$ is an arbitrary constant conveniently chosen.

For example, for the power attrition-rate coefficients (5), Theorem 2 tells us that we can transform (9) into an equation with constant coefficients only when $m=n$ or $m+n+2=0$.

\section{POWER ATTRITION-RATE COEFFICIENTS}

In this case the attrition-rate coefficients are given by (5). There are two cases to be considered, depending on whether (9) with (5) can be transformed into a constant-coefficient equation (see Theorem 2 above): (1) $m+n+2 \neq 0$, and (2) $m+n+2=0$.

Case 1. $m+n+2 \neq 0$

In order for solution methods (either successive approximations or infinite series methods) to be applicable, we must further impose the following restrictions: (a) for $C=0$, we must have $m>-1$ and $n>-1$, while (b) for $C>0$, we must have $m+n+2 \neq 0$. Only the former case was considered in reference 38 . The theory presented in the previous section is essential for extending these results to the case in which $C>0$.

From results given in reference 38 , it follows that a fundamental system of solutions to (9) with attrition-rate coefficients $(5)$ is given by

$$
x_{1}(t)=\Gamma(q)\left[\left(k_{a} k_{b}\right)^{\frac{1}{2}} / 2 s\right]^{p}(t+C)^{(m+1) / 2} I_{-p}\left[\left(k_{a} k_{b}\right)^{\frac{1}{2}}(t+C)^{s} / s\right],
$$

and

$$
x_{2}(t)=\Gamma(p)\left[\left(k_{a} k_{b}\right)^{\frac{1}{2}} / 2 s\right]^{q}(t+C)^{(m+1) / 2} I_{p}\left[\left(k_{a} k_{b}\right)^{\frac{1}{2}}(t+C)^{s} / s\right],
$$

where $I_{p}$ denotes the modified Bessel function of the first kind of order $p$, $p=(m+1) /(m+n+2), p+q=1$, and $2 s=m+n+2$, with similar results holding for $\left\{y_{1}(t), y_{2}(t)\right\}$. The above Lanchester functions, of course, satisfy all the properties given in Table I. The $X$ force level, $x(t)$, then is given by (18). 
To emphasize the dependence on the parameter $C$ it is sometimes convenient to write, for example, $x_{1}(t)=x_{1}(t ; C)$. For $a(t)$ and $b(t)$ both not constant, the only finite singularity of the $X$ and $Y$ force-level equations with the power attrition-rate coefficients $(5)$ is $t=-C$ so that $t_{0}=-C$. We note that then $x_{1}(t=0 ; C=0)=y_{1}(t=0 ; C=0)=1$ and $x_{2}(t=0 ; C=0)$ $=y_{2}(t=0 ; C=0)=0$; hence (18) with these particular fundamental systems reduces to $(20)$ when $C=0$. By Theorem 1 no such simplification of (18) occurs for $C>0$ unless $m=n$.

The above representations (22) and (23) of fundamental systems of solutions to the $X$ and $Y$ force-level equations are not particularly convenient. For example, for $m, n>-1$ we have $0<p, q<1$, and $p+q=1$, and tabulations only exist of the modified Bessel function of the first kind of fractional order $\nu$ for a restrictive set of values [i.e., $\nu= \pm 1 / 4, \pm 1 / 3, \pm 2 / 3, \pm 3 / 4$ (see reference 1)]. This Bessel function reduces to other tabulated forms (i.e., hyberbolic functions) for $\nu= \pm 1 / 2$. Therefore, it is more useful to express $x_{1}(t)$, $x_{2}(t), y_{1}(t)$, and $y_{2}(t)$ in the form of infinite series and to consider the resulting transcendental functions as entities in their own right. Thus, it is convenient to define the following Lanchester functions for power attritionrate coefficients (referred to as power Lanchester functions).

$$
\begin{gathered}
\left.u_{m, n}(t)=\Gamma(q) \sum_{k=0}^{k=\infty}\left[\left(k_{a} k_{b}\right)^{\frac{1}{2}} / m+n+2\right)\right]^{2 k} t^{k(m+n+2)} / \\
{[k ! \Gamma(k+q)],} \\
v_{m, n}(t)=\Gamma(p) \sum_{k=0}^{k=\infty}\left[\left(k_{a} k_{b}\right)^{\frac{1}{2}} /(m+n+2)\right]^{2 k+1} t^{k(m+n+2)+m+1} / \\
{[k ! \Gamma(k+1+p)],} \\
U_{m, n}(t)=\Gamma(p) \sum_{k=0}^{k=\infty}\left[\left(k_{a} k_{b}\right)^{\frac{1}{2}} /(m+n+2)\right]^{2 k} t^{k(m+n+2)} / \\
{[k ! \Gamma(k+p)],} \\
V_{m, n}(t)=\Gamma(q) \sum_{k=0}^{k=\infty}\left[\left(k_{a} k_{b}\right)^{\frac{1}{2}} /(m+n+2)\right]^{2 k+1} t^{k(m+n+2)+n+1} / \\
{[k ! \Gamma(k+1+q)] .}
\end{gathered}
$$

The power Lanchester functions $u_{m, n}, v_{m, n}, U_{m, n}$, and $V_{m, n}$ have the properties shown in Table II. For reasons to be explained below we will refer to $u_{m, n}(t)$ and $U_{m, n}(t)$ and similarly $v_{m, n}(t)$ and $V_{m, n}(t)$ as complementary power Lanchester functions. Property 5 of Table II follows from properties 6 and 7 for $m, n>-1$. We show below that it holds for all values of $m$ and $n$. Thus, for $C \geqq 0$, with $m$ and $n$ subject to the restrictions given in the paragraph immediately preceding equation (22), the solution (18) to (9) with the power attrition-rate coefficients (5) may then be written in terms of the power Lanchester functions as

$$
\begin{aligned}
x(t)=x_{0}\{ & \left.U_{m, n}(C) u_{m, n}(t+C)-V_{m, n}(C) v_{m, n}(t+C)\right\} \\
& -y_{0}\left(k_{a} / k_{b}\right)^{\frac{1}{2}}\left\{u_{m, n}(C) v_{m, n}(t+C)-u_{m, n}(t+C) v_{m, n}(C)\right\},
\end{aligned}
$$

and similarly for $y(t)$. From (28) we see that the methods of reference 38 would become hopelessly bogged down in details for $C>0$. 
TABLE II

Properties of the Power Lanchester Functions $u_{m, n}, v_{m, n}, U_{m, n}$, AND $V_{m, n}$

\begin{tabular}{lr}
\hline 1. & $d u_{m, n} / d t=\left(k_{b} / k_{a}\right)^{2}\left[k_{a} t^{m}\right] V_{m, n}(t)$ \\
2. & $d v_{m, n} / d t=\left(k_{b} / k_{a}\right)^{2}\left[k_{a} t^{m}\right] U_{m, n}(t)$ \\
3. & $d U_{m, n} / d t=\left(k_{a} / k_{b}\right)^{t}\left[k_{b} t^{n}\right] v_{m, n}(t)$ \\
4. & $d V_{m, n} / d t=\left(k_{a} / k_{b}\right)^{4}\left[k_{b} t^{n}\right] u_{m, n}(t) \quad \forall t$ \\
5. & $u_{m, n}(t) U_{m, n}(t)-v_{m, n}(t) V_{m, n}(t)=1 \quad \forall t$ \\
6. & $u_{m, n}(t=0)=U_{m, n}(t=0)=1$ \\
7. & $v_{m, n}(t=0)=V_{m, n}(t=0)=0$ \\
8. & $\left.u_{m, m}(t)=U_{m, m}(t)=\cosh \left[\left(k_{a} k_{b}\right)\right)^{2} t^{m+1} /(m+1)\right]$ \\
9. & $v_{m, m}(t)=V_{m, m}(t)=\sinh \left[\left(k_{a} k_{b}\right)^{2} t^{m+1} /(m+1)\right]$ \\
\hline
\end{tabular}

Note: Properties 6 and 7 hold only for $m, n>-1$.

For computational reasons it is convenient to introduce the auxiliary power Lanchester functions [also referred to as the Lanchester-CliffordSchläfli functions, or LCS functions (see Note 10)], which are defined for $\nu \neq 0,-1,-2,-3, \cdots$

and

$$
F_{\nu}(x)=\sum_{k=0}^{k=\infty}(x / 2)^{2 k} /\left[k ! \Pi_{j=0}^{k-1}(j+\nu)\right],
$$

$$
G_{\nu}(x)=\sum_{k=0}^{k=\infty}(x / 2)^{2 k+1} /\left[k ! \prod_{j=0}^{j=k}(j+\nu)\right],
$$

where we have adopted the convention that $\Pi_{j=M}^{j=N} f_{j}=1$ for $N<M$. The LCS functions possess the properties shown in Table III. Considering $Q(x)=F_{p}(x) F_{q}(x)-G_{p}(x) G_{q}(x)$, where $p+q=1$ and $p, q \neq 0,-1,-2, \cdots$, one may easily prove property 3 of Table III by observing that $d Q / d x=0 \forall x$ and using properties 4 and 5 .

The power Lanchester functions $u_{m, n}, v_{m, n}, U_{m, n}$, and $V_{m, n}$ may be expressed in terms of the LCS functions as follows:

$$
u_{m, n}(t)=F_{q}[\psi(t)]
$$

\section{TABLE III}

Properties of the LCS Functions $F_{\nu}(x)$ and $G_{\nu}(x)$.

\begin{aligned} & \hline 1.$d F_{\nu} / d x=G_{\nu}(x) \\ & 2. d G_{\nu} / d x=F_{\nu}(x)-\{(2 \nu-1) / x\} G_{\nu}(x) \\ & 3. F_{p}(x) F_{q}(x)-G_{p}(x) G_{q}(x)=1 \quad \forall x \\ &$ where $p+q=1$ and $p, q \neq 0,-1,-2,-3, \cdots \\ & 4. F_{\nu}(x=0)=1 \\ & 5. G_{\nu}(x=0)=0 \\ & 6. \lim _{x \rightarrow 0}\left\{G_{\nu}(x) / x\right\}=1 /(2 \nu) \\ & 7. d F_{\nu} / d x(x=0)=0 \\ & 8. d G_{\nu} / d x(x=0)=1 /(2 \nu) \\ & 9. F_{1 / 2}(x)=\cosh x \\ & 10. G_{1 / 2}(x)=\sinh x \\ &$\hline\end{aligned}




$$
\begin{aligned}
v_{m, n}(t) & =t^{(m-n) / 2} G_{p}[\psi(t)], \\
U_{m, n}(t) & =F_{p}[\psi(t)], \\
V_{m, n}(t) & =t^{(n-m) / 2} G_{q}[\psi(t)],
\end{aligned}
$$

where $\psi(t)=\left(\left[k_{a} t^{m}\right]\left[k_{b} t^{n}\right]\right)^{\frac{1}{2}} t /[(m+n+2) / 2]$. Noting that $u_{m, n}(t) U_{m, n}(t)-$ $v_{m, n}(t) V_{m, n}(t)=F_{p}[\psi(t)] F_{q}[\psi(t)]-G_{p}[\psi(t)] G_{q}[\psi(t)]$, we see that property 5 of Table II holds for all values of $m$ and $n$ and follows from property 3 of Table III. Also, $u_{m, n}(t)$ and $U_{m, n}(t)$ have been called complementary power Lanchester functions because of the above relations, the fact that $p+q=1$, and property 3 of Table III. Consequently, (28) may now be written as

$$
\begin{array}{r}
x(t)=x_{0}\left\{F_{p}[\theta(0)] F_{q}[\theta(t)]-(1+t / C)^{(m-n) / 2} G_{q}[\theta(0)] G_{p}[\theta(t)]\right\} \\
-y_{0}\{a(t=0) / b(t=0)\}^{\frac{1}{2}}\left\{(1+t / C)^{(m-n) / 2} F_{q}[\theta(0)] G_{p}[\theta(t)]\right. \\
\left.-G_{p}[\theta(0)] F_{q}[\theta(t)]\right\},
\end{array}
$$

where $p=(m+1) /(m+n+2), \quad q=1-p, \quad$ and $\theta(t)=[a(t) b(t)]^{\frac{1}{2}}(t+C) /$ $[(m+n+2) / 2]$. Introduction of the LCS functions thus sheds light on the parametric dependence of solutions: there are two exponent parameters, $p$ and $(m-n) / 2$, and an 'intensity' parameter, $\theta(t)$, as well as the relative effectiveness parameter $\left(k_{a} / k_{b}\right)^{\frac{1}{2}}$. We observe that average 'intensity' $\overline{[a(t) b(t)]^{\frac{1}{2}}}$ is related to $\theta(t)=\theta(t ; C)$ by $\left.\overline{[a(t) b(t)}\right]^{\frac{1}{2}} t=\theta(t ; C)-\theta(t=0 ; C)$. Without an algebraic addition theorem, however, this does not lead to simplification of (35). We do find, though, that for $C=0$ with $m, n>-1$ we have $[\overline{a(t) b(t)}]^{\frac{1}{2}} t=\theta(t ; C=0)$, and (35) may be written like (21) as

$$
x(t)=x_{0} F_{q}\left([\overline{a(t) b(t)}]^{\frac{1}{2}} t\right)-y_{0}[a(t) / b(t)]^{\frac{1}{2}} G_{p}\left([\overline{a(t) b(t)}]^{\frac{1}{2}} t\right) .
$$

It may be shown that $\lim _{t \rightarrow 0}[a(t) / b(t)]^{\frac{1}{2}} G_{p}\left([\overline{a(t) b(t)}]^{\frac{1}{2}} t\right)=0$.

Considering (35) and the above, we see that there is an interesting way (with some similarities with the constant coefficient situation) of thinking about combat between two homogeneous forces described by (4) and (5): the course of combat is determined by average combat 'intensity' and how it changes over time as well as the initial force levels, $x_{0}$ and $y_{0}$, and $[a(t=$ $0) / b(t=0)]^{\frac{1}{2}}$.

Case 2. $m+n+2=0$ and $C>0$

It should first be noted that the solution obtained for Case 1 becomes indeterminate [consider, for example, what happens to $u_{m, n}(t)$ as defined by (24)]. To solve the Fuchsian equation (see pp. 108-127 of reference 27) which arises from $(9)$ with $(5)$ when $m+n+2=0$, we make the substitution $u=\ln (t+C)$ to transform (9) into an equation with constant coefficients. Solving this equation and transforming back to the original independent variable $t$, we find that both members of the resulting fundamental 
system of solutions are not bounded at $t=t_{0}=-C$ (see pp. 148-150 of reference 29), and we consequently cannot impose (16). To determine a fundamental system uniquely, we take $x_{1}\left(t=t_{0}\right)$ to be unbounded and specify the conditions $x_{1}(t=0)=1,(11),(12)$, and (14) (see Section 4). The corresponding fundamental system (with the properties given in Table I) for (18) is given by

$$
x_{1}(t)=([t+C] / C)^{\alpha_{-}}, \quad \text { and } \quad x_{2}(t)=\left\{C^{-(n+1)} / 2 \gamma\right\}\left(\alpha_{-} \beta_{-}\right)^{\frac{1}{2}}([t+C] / C)^{\alpha_{+}},
$$

and similarly for $\left\{y_{1}(t), y_{2}(t)\right\}$, where $\gamma=\left\{k_{a} k_{b}+[(m+1) / 2]^{2}\right\}^{\frac{1}{2}}, \alpha_{+}=\gamma+$ $(m+1) / 2=-\beta_{-}$, and $\alpha_{-}=-\gamma+(m+1) / 2=-\beta_{+}$. In this case (18) becomes

$$
\begin{aligned}
& x(t)=x_{0}\left\{[1 /(2 \gamma)]\left[\beta_{+}([t+C] / C)^{\alpha_{+}}-\beta_{-}([t+C] / C)^{\alpha_{-}}\right\}\right. \\
& \quad-y_{0}\left(k_{a} / k_{b}\right)^{\frac{1}{2}}\left\{\left[\left(k_{a} k_{b}\right)^{\frac{1}{2}} C^{m+1} /(2 \gamma)\right]\left[([t+C] / C)^{\alpha_{+}}-([t+C] / C)^{\alpha_{-}}\right]\right\},
\end{aligned}
$$

which may also be written in the more convenient form

$$
\begin{gathered}
x(t)=\left([t+C] / C^{(m+1) / 2}\left\{x_{0}[\cosh [\gamma \ln ([t+C] / C)]\right.\right. \\
-[(m+1) /(2 \gamma)] \sinh (\gamma[t+C] / C)] \\
\left.-y_{0}\left(k_{a} / k_{b}\right)^{\frac{1}{2}}\left[\left(C^{m+1}\left(k_{a} k_{b}\right)^{\frac{1}{2}} / \gamma\right) \sinh \{\gamma \ln ([t+C] / C)\}\right]\right\} .
\end{gathered}
$$

Although the above solutions appear complicated, they are readily evaluated with the help of a portable electronic calculator such as is commercially available today. In fact, such a calculator can even be 'programmed' to facilitate parametric analyses.

\section{WEAPON SYSTEMS WITH DIFFERENT EFFECTIVE RANGES: LINEAR ATTRITION-RATE COEFFICIENTS}

Another interesting situation in which to apply our general theory is that of combat between two homogeneous forces that use weapons with different effective ranges. We consider the example previously examined by Bonder and Farrell ${ }^{[10]}$ and Taylor ${ }^{[38]}$ of a constant-speed attack of a mobile force against a static defense. The weapon systems of the two sides have different effective ranges, and the lethality of each side's fire depends linearly upon range. We assume that the opening range of battle, $R_{0}$, satisfies $R_{0} \leqq$ minimum $\left(R_{\alpha}, R_{\beta}\right)$, where $R_{\alpha}$ denotes the maximum effective range of the $Y$ system. The Lanchester attrition-rate coefficients for such a battle may be written as (6). The parameter $C$ is related to the opening range of battle in comparison with minimum $\left(R_{\alpha}, R_{\beta}\right)$, whereas $A$ reflects the difference in maximum effective ranges [see (51) in Section 7]. Figure 1 shows the range dependencies of these Lanchester attrition-rate coefficients and the opening range of battle.

From results given in reference 38 , it follows that a fundamental system of solutions to (9) (which has the properties shown in Table I) with the 
attrition-rate coefficients (6) (and also one for the $Y$ force-level equation) is given by

$$
\begin{gathered}
x_{1}(t)=f(t+C), \quad x_{2}(t)=g(t+C), \\
y_{1}(t)=F(t+C), \quad \text { and } y_{2}(t)=G(t+C), \\
f(t)=\sum_{n=0}^{n=\infty}\left\{\left[\left(k_{a} k_{b}\right)^{\frac{1}{2}} / 2\right]^{2 n} /(2 n) !\right\} \sum_{k=0}^{k=n} B_{n}{ }^{k} A^{k} t^{4 n-k}, \\
g(t)=\sum_{n=0}^{n=\infty}\left\{\left[\left(k_{a} k_{b}\right)^{\frac{1}{2}} / 2\right]^{2 n+1} /(2 n+1) !\right\} \sum_{k=0}^{k=n} C_{n}^{k} A^{k} t^{4 n+2-k},
\end{gathered}
$$

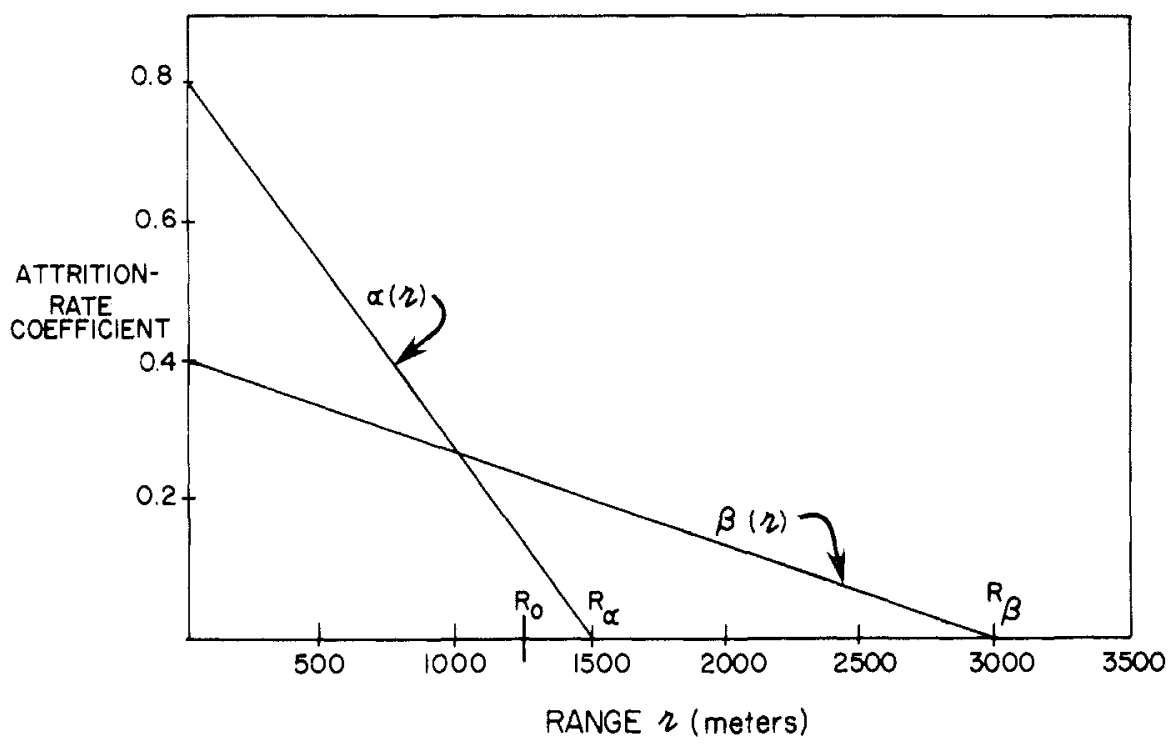

Fig. 1. Linear attrition-rate coefficients for weapon systems with different effective ranges. [Notes: 1. The maximum effective ranges of the two weapon systems are denoted as $R_{\alpha}$ and $R_{\beta}$. 2. The opening range of battle is denoted as $R_{0}$ and (as shown) $R_{0}<$ minimum $\left(R_{\alpha}, R_{\beta}\right)$.]

$$
\begin{aligned}
& F(t)=\sum_{n=0}^{n=\infty}\left\{\left[\left(k_{a} k_{b}\right)^{\frac{1}{2}} / 2\right]^{2 n} /(2 n) !\right\} \sum_{k=0}^{k=n} D_{n}{ }^{k} A^{k} t^{4 n-k}, \\
& G(t)=\sum_{n=0}^{n=\infty}\left\{\left[\left(k_{a} k_{b}\right)^{\frac{1}{2}} / 2\right]^{2 n+1} /(2 n+1) !\right\} \sum_{k=0}^{n+1} E_{n}{ }^{k} A^{k} t^{4 n+2-k} .
\end{aligned}
$$

The coefficients $B_{n}{ }^{k}$ and $C_{n}{ }^{k}$ are given in reference 38 , while the coefficients $D_{n}{ }^{k}$ and $E_{n}{ }^{k}$ are given by $D_{0}{ }^{0}=1$ and for $n>0$

$$
D_{n}{ }^{k}=\left\{\begin{array}{cc}
1, & \text { for } k=0, \\
{[4 n(4 n-2) /(4 n-k)]\left\{\left[D_{n-1}^{k} /(4 n-k-2)\right]\right.} & \\
\left.+\left[D_{n-1}^{k-1} /(4 n-k-1)\right]\right\}, & \text { for } 1 \leqq k \leqq n-1, \\
(4 / 3)[(4 n-2) /(3 n-1)] D_{n-1}^{n-1}, & \text { for } k=n ;
\end{array}\right.
$$


and $E_{0}{ }^{0}=1, E_{0}{ }^{1}=2$, and for $n>0$

$$
E_{n}{ }^{k}=\left\{\begin{aligned}
1, & \text { for } k=0, \\
{[4 n(4 n+2) /(4 n-k+2)]\left\{\left[E_{n-1}^{k} /(4 n-k)\right]\right.} & \\
\left.+\left[E_{n-1}^{k-1} /(4 n-k+1)\right]\right\}, & \text { for } 1 \leqq k \leqq n, \\
(4 / 3)[(4 n+2) /(3 n+1)] E_{n-1}^{n}, & \text { for } k=n+1,
\end{aligned}\right.
$$

where we have adopted the convention that $0^{0}=1$. We note that $f(t=0)=$ $F(t=0)=1$ and $g(t=0)=G(t=0)=0$. Motivated by their origin within the context of a constant-speed attack of mobile forces as first studied by Bonder, ${ }^{[5,7,10]}$ we will refer to the above Lanchester functions for the linear attrition-rate coefficients with nonconstant ratio (6) as offset linear Lanchester functions. We use the word 'offset' to denote a nonzero difference between the maximum effective ranges of the two systems (consider Fig. 1).

To emphasize the dependence on the parameter $A$, it is sometimes convenient to write, for example, $f(t)=f(t ; A)$. We note that $f(t ; A=0)=$ $F(t ; A=0)=\cosh \left[\left(k_{a} k_{b}\right)^{\frac{1}{2}} t^{2} / 2\right]$ and $g(t ; A=0)=G(t ; A=0)=\sinh \left[\left(k_{a} k_{b}\right)^{\frac{1}{2}} t^{2} / 2\right]$. For $A=0$, the fundamental property $f(t) F(t)-g(t) G(t)=1$ of these offset linear Lanchester functions becomes a well known property of the hyperbolic functions.

As was the case for the power attrition-rate coefficients of the previous section, for computational reasons it is convenient to introduce the following auxiliary offset linear Lanchester functions (depending on two parameters):

$$
\begin{aligned}
h(\lambda, \mu) & =\sum_{n=0}^{n=\infty}\left\{\lambda^{2 n} /(2 n) !\right\} \sum_{k=0}^{k=n} B_{n}{ }^{k} \mu^{k}, \\
w(\lambda, \mu) & =\sum_{n=0}^{n=\infty}\left\{\lambda^{2 n+1} /(2 n+1) !\right\} \sum_{k=0}^{k=n} C_{n}{ }^{k} \mu^{k}, \\
H(\lambda, \mu) & =\sum_{n=0}^{n=\infty}\left\{\lambda^{2 n} /(2 n) !\right\} \sum_{k=0}^{k=n} D_{n}{ }^{k} \mu^{k}, \\
W(\lambda, \mu) & =\sum_{n=0}^{n=\infty}\left\{\lambda^{2 n+1} /(2 n+1) !\right\} \sum_{k=0}^{n+1} E_{n}{ }^{k} \mu^{k} .
\end{aligned}
$$

The auxiliary offset linear Lanchester functions possess the following properties:

$$
\begin{array}{ll}
\text { 1. } & h(\lambda, \mu) H(\lambda, \mu)-w(\lambda, \mu) W(\lambda, \mu)=1 \forall \lambda, \mu, \\
\text { 2. } & h(\lambda, 0)=H(\lambda, 0)=\cosh \lambda, \\
\text { 3. } & w(\lambda, 0)=W(\lambda, 0)=\sinh \lambda .
\end{array}
$$

Using the auxiliary offset linear Lanchester functions, we may write the $X$ force level as

$$
\begin{aligned}
x(t)=x_{0} & \{H[\theta(0), \delta(0)] h[\theta(t), \delta(t)]-W[\theta(0), \delta(0)] w[\theta(t), \delta(t)]\} \\
& -y_{0}\left(k_{a} / k_{b}\right)^{\frac{1}{2}}\{h[\theta(0), \delta(0)] w[\theta(t), \delta(t)] \\
& -w[\theta(0), \delta(0)] h[\theta(t), \delta(t)]\}
\end{aligned}
$$


where $\delta(t)=A /(t+C)$ and $\theta(t)=\left(k_{a} k_{b}\right)^{\frac{1}{2}}(t+C)^{2} / 2$. The expression $\delta(t)$ reflects how much the above Lanchester functions deviate from the hyperbolic functions.

\section{SOME NUMERICAL EXAMPLES}

In this section we examine three numerical examples that illustrate possible use of some of our new results. These examples are extensions of the ones given previously in reference 38 by having the opening range of battle be less than the minimum of the two maximum effective ranges for the two weapon systems. They are motivated by the work of Bonder ${ }^{[5,7,10]}$ on the value of range capabilities and mobility for weapon systems in combat described by Lanchester-type equations of modern warfare. The modelling context of these examples is that of weapon systems with (a) different range dependencies of lethality of each side's fire, but the same maximum effective range, and (b) linear attrition-rate coefficients, but different effective ranges.

As in reference 38 we consider a constant-speed attack on a static defensive position with the combat dynamics described by

$$
\begin{aligned}
& d x / d t=-\alpha(r) y=-\alpha_{0}\left(1-r / R_{\alpha}\right)^{m} y, \\
& d y / d t=-\beta(r) x=-\beta_{0}\left(1-r / R_{\beta}\right)^{n} x,
\end{aligned}
$$

where $R_{\alpha}$ and $R_{\beta}$ denote the maximum effective ranges of the $Y$ and $X$ weapon systems, respectively (i.e., $\alpha(r)=0$ for $r>R_{\alpha}$ ). The reader should consider (49) to be (4) with the attrition-rate coefficients (5) or (6) (as is appropriate) expressed in terms of range (i.e., force separation). Range is related to time by $r(t)=R_{0}-v t$, where $R_{0}$ denotes the opening range of battle. Several range dependencies for an attrition-rate coefficient are shown in Fig. 2, and an opening range less than the weapon system's maximum effective range is indicated. The parameters of the attrition-rate coefficients $\alpha[r(t)]$ and $\beta[r(t)]$ in (48) are readily related to those of $a(t)$ and $b(t)$ in (5) and (6). For (5) we have

$$
k_{a}=\alpha_{0}\left(v / R_{\alpha}\right)^{m}, \quad k_{b}=\beta_{0}\left(v / R_{\beta}\right)^{n}, \quad \text { and } C=\left(R_{\alpha}-R_{0}\right) / v,
$$

where $R_{\alpha}=R_{\beta} \geqq R_{0}$, while for (6) we have

$$
k_{u}=\alpha_{0} v / R_{\alpha}, k_{b}=\beta_{0} v / R_{\beta}, A=\left(R_{\beta}-R_{\alpha}\right) / v \text {, and } C=\left(R_{\alpha}-R_{0}\right) / v \text {, }
$$

where $R_{\beta} \geqq R_{\alpha} \geqq R_{0}$.

Numerical results are shown in Figs. 3, 4, and 5. The force-level trajectories have been generated by a digital computer program using the auxiliary Lanchester functions [i.e., $F_{\nu}$ and $G_{\nu}$ for (35) and $h, w, H$, and $W$ for (48)]. These functions are particularly convenient for such digital computer work. The computer routines were checked against the numerical results given previously in reference 38 . In this work we have taken the 
opening range of battle $R_{0}<$ minimum $\left(R_{\alpha}, R_{\beta}\right)$. Numerical values for battle parameters (except those for $R_{0}, R_{\alpha}$, and $R_{\beta}$ ) are the same as those used for the examples considered in reference 38 .

For Figs. 3 and 4 both weapon systems have the same maximum effective range (i.e., $R_{\alpha}=R_{\beta}$ ). As done for the plots in reference 38 , we have held $\alpha_{0}=\alpha(r=0)$ and $\beta_{0}$ constant and have varied the exponents $m$ and $n$, which

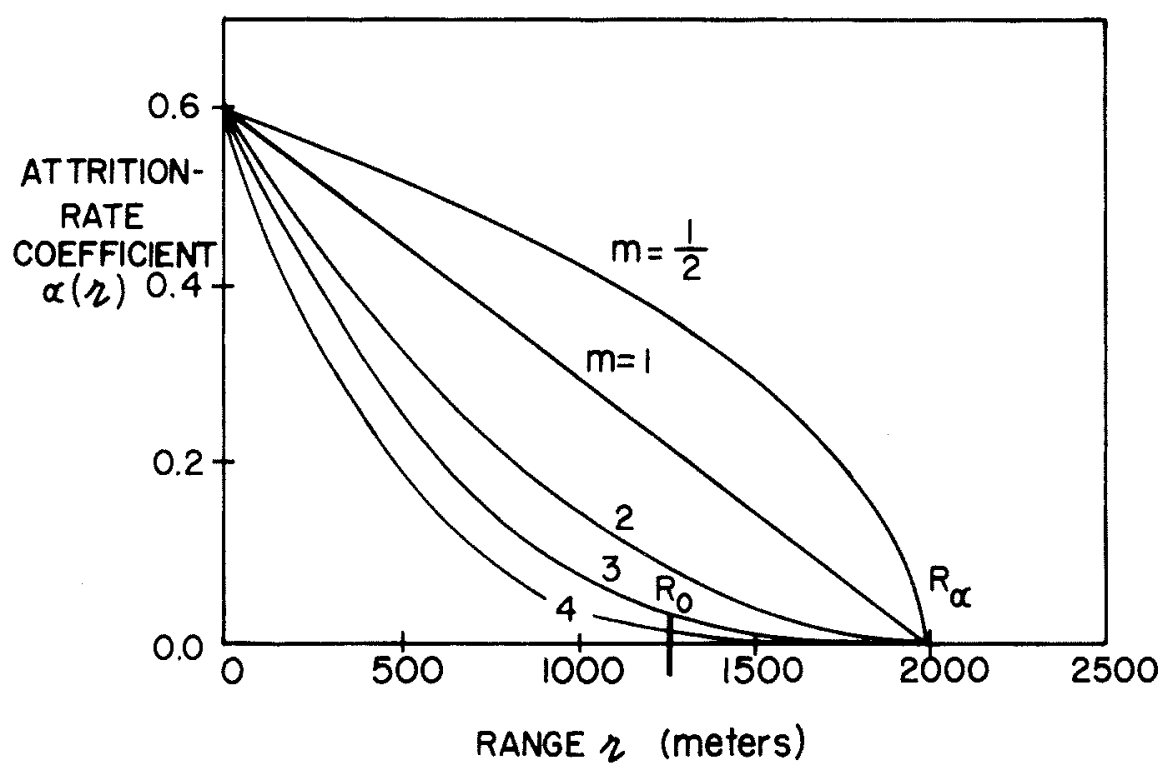

Fig. 2. Dependence of the attrition-rate coefficient $\alpha(r)$ on the exponent $m$ for constant maximum effective range of the weapon system and constant kill capability at zero range. [Notes: 1. The maximum effective range of the system is denoted $R_{\alpha}=2000$ meters. 2. $\alpha(r=0)=\alpha_{0}=0.6 X$ casualties/(unit time $\times$ number of $Y$ units) denotes the $Y$ force weapon system kill rate at zero force separation (range). 3. The opening range of battle is denoted as $R_{0}=1250$ meters and (as shown) $R_{0}<R_{\alpha}$ ].

control the range dependencies of $\alpha(r)$ and $\beta(r)$. With the exception of $R_{0}$, all the battle parameters for the curves shown in Fig. 3 are the same as those used for the corresponding example previously considered in reference 38 (i.e., $R_{\alpha}=R_{\beta}=2000$ meters). Consequently, corresponding force-level trajectories are similar, with greater 'separation' shown here between curves. In Fig. 3 with the opening range of battle $R_{0}=1250$ meters, the curves corresponding to the constant-coefficient case (i.e., $m=n=0$ ) are exactly the same (for the same time intervals) as those shown in reference 38 with $R_{0}=2000$ meters. Other battle trajectories with $m, n>0$ decay faster than they did in reference 38 because the 'intensity' of combat is greater; i.e., 


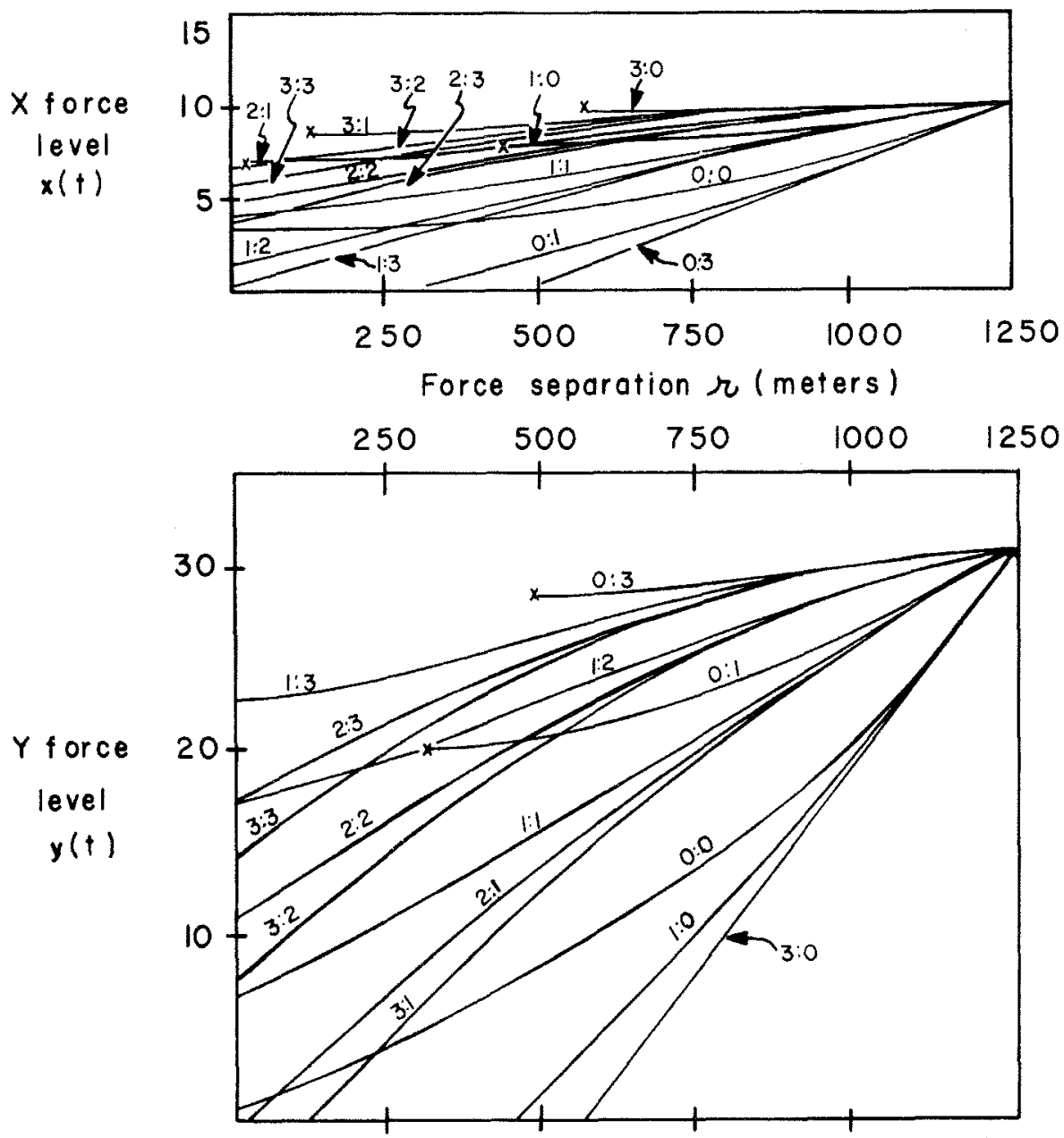

Fig. 3. Force-level trajectories of $X$ and $Y$ forces for various combinations of the exponents $m$ and $n$ in the power attrition-rate coefficients for $R_{0}=1250$ meters, $R_{\alpha}=R_{\beta}=2000$ meters, $\alpha_{0}=0.06 X$ casualties $/$ (minutes $Y$ unit), $\beta_{0}=0.6 Y$ casualties/(minutes $X$ unit), $v=5 \mathrm{mph}, x_{0}=10$, and $y_{0}=30$. The exponent combinations are denoted as $m: n$ in the figure, and the symbol $\times$ denotes the end of a force-level trajectory due to the annihilation of the enemy force.

as a function of time the attrition-rate coefficients are larger here than in reference 38 (see Fig. 2).

As noted in references 5 and 38 , knowledge of the range or time dependence of weapon-system kill capability is essential for forecasting the battle's outcome from the initial trend of battle. For example, compare in Fig. 3 the outcomes for curves denoted as 1:0, 1:1, and 1:2. Clark ${ }^{[12]}$ 
has developed methodology for estimating such capability from the output of a high-resolution Monte Carlo combat simulation. Figure 4 shows similar battle curves for the same parameter values, except that $R_{\alpha}=R_{\beta}=1500$ meters. Observing that for $m \geqq 1$ we have $\alpha\left(r ; R_{\alpha}\right)<\alpha\left(r ; \bar{R}_{\alpha}\right) \Leftrightarrow R_{\alpha}<\bar{R}_{\alpha}$,

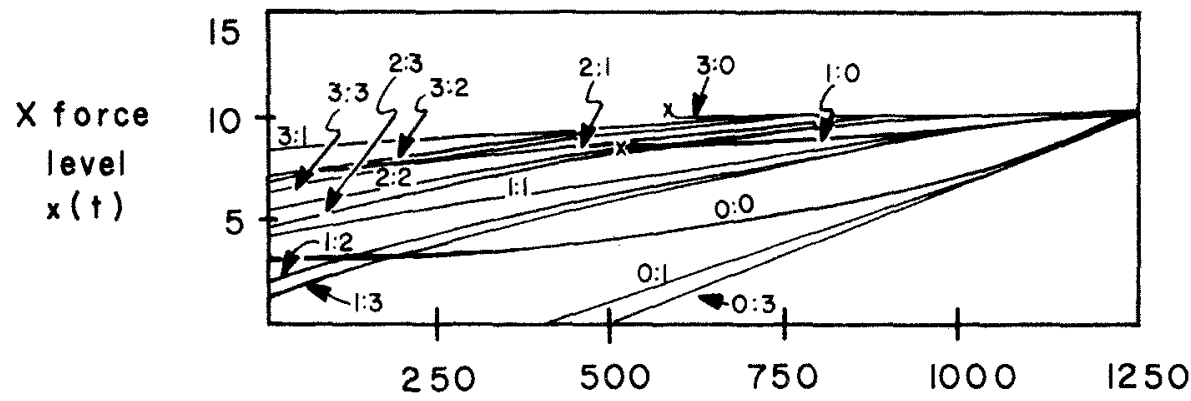

Force separation $r$ (meters)

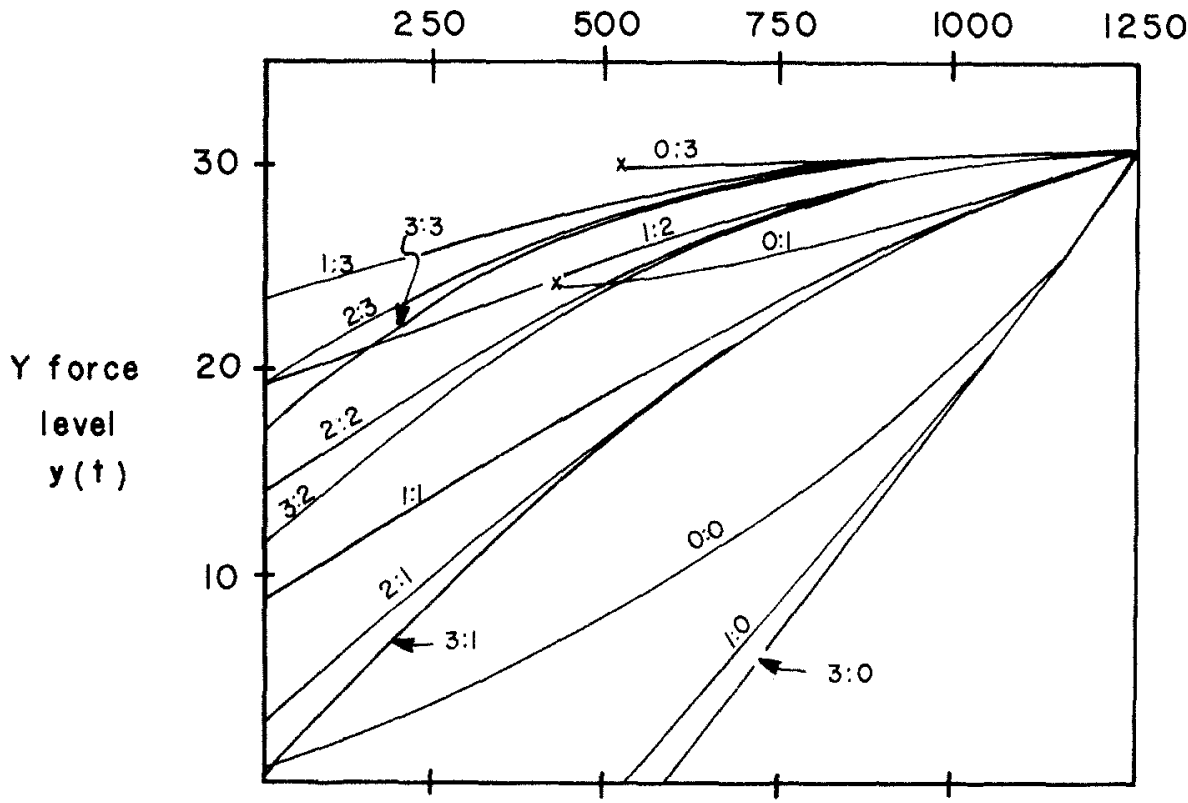

Fig. 4. Force-level trajectories of $X$ and $Y$ forces for various combinations of the exponents $m$ and $n$ in the power attrition-rate coefficients for the same parameter values chosen for Fig. 3 except that $R_{\alpha}=R_{\beta}=1500$ meters. The symbol conventions are also the same as in Fig. 3.

we may consider that the combat is less 'intense' for such pairs of battle trajectories in Fig. 4 than for those shown in Fig. 3.

We consider next numerical results for the linear attrition-rate coefficients with nonconstant ratio (6). In Fig. 5 we show the effect of increas- 
ing the effective range of the defender's weapons. (The $X$ force may be considered to be the defender.) For these computations, using (48) and the auxiliary offset linear Lanchester functions, we have held the opening range of battle constant at $R_{0}=1250$ meters and the maximum effective

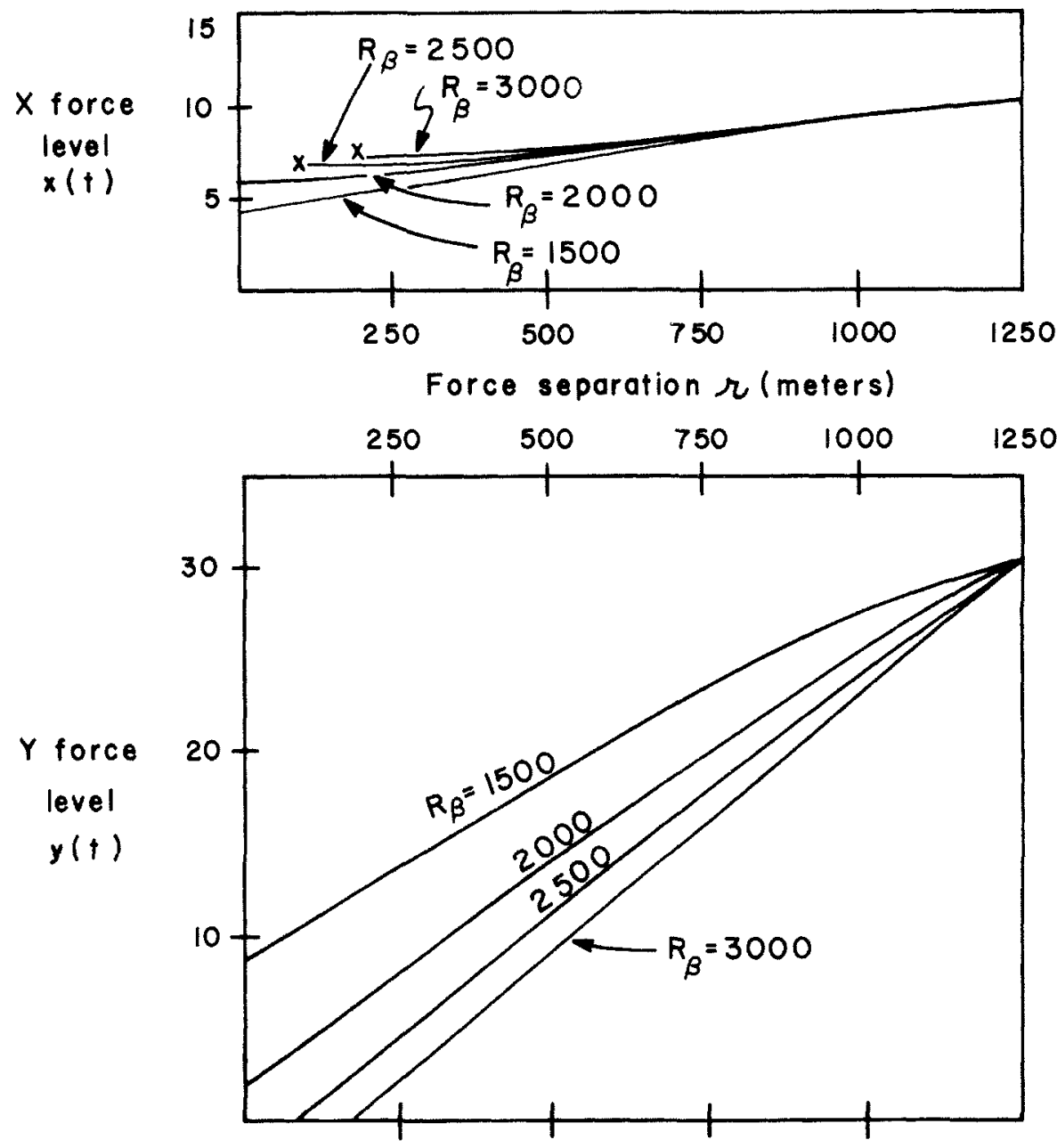

Fig. 5. Force-level trajectories of $X$ and $Y$ forces for various effective ranges $R_{\beta}$ of the $X$ force weapons with linear attrition-rate coefficients for $R_{\alpha}=1500$ meters and the same values of the other parameters listed in the legend of Fig. 3 . The symbol $\times$ has the same meaning as in this figure.

range of the $Y$ weapon constant at $R_{\alpha}=1500$ meters. As in reference 38 , both attrition-rate coefficients depend linearly on range [i.e., $m=n=1$ in (49)], $\alpha_{0}$ and $\beta_{0}$ have been held constant, and $R_{\beta}$ has been varied. As shown in Fig. 5, we quantiatively see the benefit from increasing the long-range 
kill capability of the defender's weapons. (See pp. 296-367 of reference 10 for extensive parametric studies based on analogue computer results. The analytic results given here may be used for such investigations.) It should be emphasized that although the results shown in Figs. 3, 4, and 5 are similar to those in reference 38 , our previous analytic results were limited to the case in which the opening range of battle $R_{0}=$ minimum $\left(R_{\alpha}, R_{\beta}\right)$.

\section{DEVELOPMENT OF TABULATIONS}

Tabulations, such as those given in reference 1, of the auxiliary Lanchester functions (i.e., the LCS functions and the auxiliary offset linear Lanchester functions) would lead to analysts being able to generate numerical solutions to the variable-coefficient Lanchester-type equations considered above with somewhat the same facility as for the constant-coefficient case. One may develop tabulations of the LCS functions $F_{\nu}(x)$ and $G_{\nu}(x)$ directly from their power series representations (29) and (30). In digital computer work we have found it convenient to calculate $F_{\nu}(x)$ from $F_{\nu}(x)=\sum_{k=0}^{k=\infty} f_{k}(x)$, where $f_{k}(x)$ is given recursively by $f_{k}(x)=$ $(x / 2)^{2} f_{k-1}(x) /[k(k-1+\nu)]$ for $k=1,2, \cdots$.with $f_{0}(x)=1$ and similarly for $G_{\nu}(x)$. For $m, n=0,1,2,3$ in the power attrition-rate coefficients $(5)$, one has the requirement for tabulations of $F_{\nu}(x)$ and $G_{\nu}(x)$ for $\nu=1 / 3,2 / 3$, $1 / 4,3 / 4,1 / 5,2 / 5,3 / 5,4 / 5,3 / 7$, and $4 / 7$. One may use (14) to reduce the number of series evaluations in such work. Tabulations of the auxiliary offset linear Lanchester functions are readily developed from the series (44) through (47) with, for example, $D_{n}{ }^{k}$ computed recursively. We have followed the above computational procedures in the work reported in Section 7 .

The determination of exactly which Lanchester functions should be tabulated depends on which functional forms for attrition-rate coefficients are most important in applications, and consideration of this is beyond the scope of this paper. The specific time-dependent, attrition-rate coeffcients we have considered have been suggested by Bonder's work on the range dependency of Lanchester attrition-rate coefficients (see pp. 196-200 of reference 10 ).

\section{SUMMARY}

We have presented a mathematical theory (including new general analytic results) for the solution of variable-coefficient Lanchester-type equations of 'modern warfare' for combat between two homogeneous forces. These results allow one to extend results given in references 10 and 38 that applied under rather restrictive conditions, e.g., opening range of battle equal to the minimum of the two maximum effective ranges of the weapon systems. It has been shown that in general the deterministic time histories of the $X$ and $Y$ force levels [i.e., $x(t)$ and $y(t)$ ] may be expressed in terms of four complementary Lanchester functions and not two as in the constant- 
coefficient case. The mathematical properties of these general Lanchester functions that facilitate analytic solution have been given. We have paid particular attention to the determination of the conditions on attrition-rate coefficients that allow relatively simple analytic results. We have applied these general results to two types of attrition-rate coefficients: power attrition-rate coefficients and linear attrition-rate coefficients with nonconstant ratio.

These results may be used to facilitate parametric analysis of the dynamic combat interactions between two homogeneous forces with time- or range-dependent weapon system capabilities. Such models are of particular interest in light of the work of Bonder ${ }^{[6,8]}$ and others in references 3,10 , 30 , and 31 on the prediction of Lanchester attrition-rate coefficients from weapon-system performance data and the work of Clark ${ }^{[12]}$ on the estimation of such time-dependent coefficients from Monte Carlo simulation output. Additionally, our new theoretical results may be used to solve Grubbs and Shuford's new probabilistic formulation for Lanchester combat theory [see equations (14) and (15) of reference 17]. A further discussion of applications is found in references 10 and 38 .

\section{NOTES}

1. This is due to the complexity of obtaining analytic results from stochastic formulations. The computational limitations of Monte Carlo simulation, especially high resolution or for combat between large units, are well known. The work of Clark shows (see pp. 102-103 of reference 12) the complexity of an analytic solution for even an idealized combat situation modelled as a continuous parameter Markov chain (see reference 12 for further references). Moreover, Bonder and Farrell ${ }^{[10]}$ report excellent agreement between Monte Carlo or stochastic simulation results and those for a corresponding deterministic Lanchester-type model with numerical solution generated by finite difference methods (see reference 42). Of course, verification of such models, as with any combat model is an unresolved question (see reference 9; further references are given in reference $38)$.

2. There is, moreover, far from universal agreement as to what are the significant variables that describe the combat process and can be used to predict its outcome. For some other views see references 19 and 24.

3. The influential military philosopher of the 19th century, Carl von Clausewitz (1780-1831) stated in his classic work On War (Vom Kriege), "The best Strategy is always to be very strong, first generally then at the decisive point... There is no more imperative and no simpler law for Strategy than to keep the forces concentrated," (p. 276 of reference 13).

4. We note that for any homogeneous force model of the form $d x / d t=$ $-h(t) A(x, y), d y / d t=-h(t) B(x, y)$, the transformation $\tau=\int_{0}^{t} h(s) d s$ 
(where we must place restrictions on $h(t)$ to ensure integrability) leads to the autonomous system $d x / d \tau=-A(x, y), d y / d_{\tau}=-B(x, y)$, where $A$, $B$, and $h$ are nonnegative functions of their argument(s).

5. Bonder and Farrell ${ }^{[10]}$ use range, i.e., force separation, as the independent variable in their work, while Taylor ${ }^{[88]}$ uses time, as we have done here. Moreover, the $X$ force-level equation (9) with attrition-rate coefficients (6) is apparently a differential equation that has not been encountered previously in applied mathematics. The differential equation under consideration could not be found among the 445 linear second-order equations tabulated in reference 22 or the 596 tabulated in reference 27 .

6 . One could equally well develop a generalization of the constant-coefficient solution expressed as

$$
x(t)=\left\{\left[x_{0}+y_{0}(a / b)^{\frac{1}{2}}\right] \exp \left\{-(a b)^{\frac{1}{2}} t\right\}+\left[x_{0}-y_{0}(a / b)^{\frac{1}{2}}\right] \exp \left\{(a b)^{\frac{1}{2}} t\right\}\right\} / 2 .
$$

In this case two of the general Lanchester functions would be like decreasing exponential functions (with the appropriate arguments) and the other two increasing exponential functions. We have chosen to generalize the form of (3), since it appears to be more convenient for parametric studies in which one might, for example, want to vary initial force levels or some measure of relative effectiveness. (In the constant coefficient case the latter is $a / b$.)

7. The singularities of the $X$ force-level differential equation (9) occur at the zeros and singularities of $a(t)$ and at the singularities of $a(t) b(t)$. For example, if $a(t)$ is a rational function, i.e., the ratio of two polynomials, then its logarithmic derivative, $\{1 / a(t)\} d a / d t$, has a simple pole at the zeros and poles of $a(t)$. Consequently, when $a(t)$ and $b(t)$ are both rational functions, $t_{0}$ belongs to the set of points consisting of the zeros and the poles of $a(t)$ and $b(t)$. Finally, we note that $t_{0}=-C$ for $(9)$ with the attritionrate coefficients (5) and also (6).

8. $W\left(x_{1}, x_{2}\right)$ may vanish, however, at $t_{0}$, which is a singularity of the differential equation (9) (see Note 7 above and pp. 141-142 of reference 29).

9. For a discussion of algebraic addition theorems see Chapter II of reference 18. Hancock gives the following theorem (p. 37, reference 18): a necessary and sufficient condition for a single-valued analytic function $f(z)$ to possess an algebraic addition theorem is that there exist between the function $f(z)$ and its first derivative an algebraic equation whose coefficients are independent of the argument $z$. No more comprehensive result could be found in the more recent monograph by Aczel. ${ }^{[2]}$

10. A function similar to $F_{v}(x)$ was introduced by Ludwig Schläfli in $1867^{[32]}$ and another appears in a posthumous fragment by William Kingdon Clifford (1845-1879) (see pp. 346-348 of reference 14). Greenhill ${ }^{[6]}$ suggested that such a function would be convenient for certain engineering problems, although he was severely criticized by Watson (see p. 91 of refer- 
ence 43). Since no tabulations of the above or modified Bessel functions of the first kind of fractional order (except for the restrictive set of values $\nu= \pm 1 / 4, \pm 1 / 3, \pm 1 / 2, \pm 2 / 3, \pm 3 / 4$ ) exist, it would seem appropriate to introduce the LCS functions as we have done.

\section{ACKNOWLEDGMENTS}

This research was supported by the Office of Naval Research as part of the Foundation Research Program at the Naval Postgraduate School. We wish to thank the referees for their many valuable suggestions, in particular, one referee who generously offered many helpful comments and suggested the proof of (14).

\section{REFERENCES}

1. M. Abramowitz and I. Stegun (ed.), Handbook of Mathematical Functions, National Bureau of Standards Applied Mathematics Series, No. 55, Washington, D. C., 1964.

2. J. Aczel, Lectures on Functional Equations and Their Applications, Academic Press, New York, 1966.

3. C. BARFoot, "The Lanchester Attrition-Rate Coefficient: Some Comments on Seth Bonder's Paper and a Suggested Alternate Method," Opns. Res. 17, 888-894 (1969).

4. H. Bateman, Differential Equations, Longmans, London, 1918 (reprinted by Chelsea Publishing Co., New York, 1966).

5. S. Bonder, "A Theory for Weapon System Analysis," Proc. U.S. Army Opns. Res. Symp. 4, 111-128 (1965).

6. S. BONDER, "The Lanchester Attrition-Rate Coefficient," Opns. Res. 15, 221232 (1967).

7. S. BoNDer, "A Model of Dynamic Combat," pp. IV-1 to IV-37 in Topics in Military Operations Research, The University of Michigan Engineering Summer Conferences, 21 July-1 August 1969.

8. S. Bonder, "The Mean Lanchester Attrition Rate," Opns. Res. 18, 179-181 (1970).

9. S. BONDER, "Systems Analysis: A Purely Intellectual Activity," Military Rev. 51, No. 2, 14-23 (1971).

10. S. Bonder and R. Farrell (eds.), "Development of Models for Defense Systems Planning," Report No. SRL 2147 TR 70-2 (U), Systems Research Laboratory, The University of Michigan, Ann Arbor, Michigan, Sept. 1970.

11. H. Brackney, "The Dynamics of Military Combat," Opns. Res. 7, 30-44 (1959).

12. G. Clark, The Combat Analysis Model, Ph.D. Dissertation, The Ohio State University, 1969.

13 C. von Clausewitz, On War, edited with an introduction by A. Rapoport, Penguin Books Ltd., Harmondsworth, Middlesex, England, 1968.

14. W. K. CuIrrond, Mathematical Papers, Macmillan, London, 1882 (reprinted by Chelsea Publishing Co., New York, 1968). 
15. L. Dolansky, "Present State of the Lanchester Theory of Combat," Opns. Res. 12, 344-358 (1964).

16. G. Greenhild, "The Bessel-Clifford Function and Its Applications," Phil. Mag., Series 6 38, 501-528 (1919).

17. F. Grubbs and J. Shuford, "A New Formulation of Lanchester Combat Theory," Opns. Res. 21, 926-941 (1973).

18. H. HANCOCK, Lectures on the Theory of Elliptic Functions, Wiley, New York, 1910 (reprinted by Dover Publications, Inc., New York, 1958).

19. P. HaYward, "The Measurement of Combat Effectiveness," Opns. Res. 16, 314-323 (1968).

20. E. INCE, Ordinary Differential Equations, Longmans, Green and Co., London, 1927 (reprinted by Dover Publications, Inc., New York, 1956).

21. R. IsaAcs, Differential Games, Wiley, New York, 1965.

22. E. Kaмke, Differentialgleichungen, Lösungsmethoden und Lösungen, Band 1, Gewöhnliche Differentialgleichungen, 3.Auflage, Akademische Verlagsgesellschaft, Leipzig, 1944 (reprinted by Chelsea Publishing Co., New York, 1971).

23. F. W. Lanchester, "Aircraft in Warfare: The Dawn of the Fourth Arm-No. V., The Principle of Concentration," Engineering 98, 422-423 (1914) (reprinted on pp. 2138-2148 of The World of Mathematics, Vol. IV, J. Newman (ed.), Simon and Schuster, New York, 1956).

24. B. H. Liddeld Hart, Strategy, Praeger, New York, 1967 (2nd Rev. Ed.).

25. J. McCloskey, "Of Horseless Carriages, Flying Machines, and Operations Research: A Tribute to Frederick William Lanchester (1868-1946)," Opns. Res. 4, 141-147 (1956).

26. P. Morse And G. Kimbald, Methods of Operations Research, The M.I.T. Press, Cambridge, Massachusetts, 1951.

27. G. Murphy, Ordinary Differential Equations and Their Solutions, Van NostrandReinhold, New York, 1960.

28. T. Oberbeck, "A Second Note on the Lanchester Equations," RM-13, The Rand Corp., Santa Monica, California, June 1947.

29. F. W. J. Olver, Asymptotics and Special Functions, Academic Press, New York, 1974.

30. J. Rustagi and R. Laitinen, "Moment Estimation in a Markov-Dependent Firing Distribution," Opns. Res. 18, 918-923 (1970).

31. J. Rustagi and R. Srivastava, "Parameter Estimation in a Markov Dependent Firing Distribution," Opns. Res. 16, 1222-1227 (1968).

32. L. SchläFl, "Sulle relazioni tra diversi integrali definiti che giovano ad esprimere la soluzione generale della equazione di Riccati," Ann. Mat. pura appl. $[2], 1,232-242$ (1867/68) (also pp. 85-94 in Gesammelte Mathematische Abhandlungen, Band III, Birkhäuser, Basel, 1956).

33. R. N. SNow, "Contributions to Lanchester Attrition Theory," Report RA15078, The Rand Corp., Santa Monica, California, April 1948.

34. J. TAYLOR, "Application of Differential Games to Problems of Military Conflict: Tactical Allocation Problems-Part I," Tech. Report NPS55Tw70062A, Naval Postgraduate School, Monterey, California, June 1970.

35. J. TAYLOR, "A Note on the Solution to Lanchester-Type Equations with Variable Coefficients," Opns. Res. 19, 709-712 (1971). 
36. J. TAYLOR, "Comments on 'A Note on the Solution to Lanchester-Type Equations with Variable Coefficients," "Opns. Res. 20, 1194-1195 (1972).

37. J. TAYLOR, "Lanchester-Type Models of Warfare and Optimal Control." Naval Res. Log. Quart. 21, 79-106 (1974).

38. J. TAYLOR, "Solving Lanchester-Type Equations for 'Modern Warfare' with Variable Coefficients," Opns. Res. 22, 756-770 (1974).

39. J. TAYLoR, "Survey on the Optimal Control of Lanchester-Type Attrition Processes," to appear in Proc. Symp. State-of-the-Art of Math. in Combat Models (also Tech. Report NPS55Tw74031, Naval Postgraduate School, Monterey, California, March 1974).

40. J. TAYLOR, "Target Selection in Lanchester Combat: Heterogeneous Forces and Time-Dependent Attrition-Rate Coefficients," Naval Res. Log. Quart. 21, 683-704 (1974).

41. J. Taylor and S. PARRy, "Force-Ratio Considerations for Some LanchesterType Models of Warfare," Opns. Res. 23, 522-533 (1975).

42. J Todd (ed.), Survey of Numerical Analysis, McGraw-Hill, New York, 1962.

43. G. Watson, A Treatise on the Theory of Bessel Functions, 2nd Ed., Cambridge University Press, Cambridge, 1944.

44. H. WeIss, "Lanchester-Type Models of Warefare," pp. 82-98 in Proceedings First International Conference on Operational Research, Wiley, New York, 1957. 